\title{
A Novel Synthesis of Flavanones from 2-Hydroxybenzoic Acids
}

\author{
Jae In Lee, "Mi Gung Jung, and Hye Jin Jung
}

\author{
Department of Chemistrv Plant Resources Research Institute. College of Natural Science, Duksung Women's University, \\ Seoul 132-714. Korea. "E-mail: jilee oduksung.achr \\ Received November 21. 2006
}

Key Words : Flavanones. 2'-Hydroxyacetophenones, 1-(2'-Hydroxypheny1)-1-oxo-propan-3-phenyl-3-ols. Cyclodelydration

The flavanones are mainly distributed in citrus fruits and have attracted considerable attention because they possess antioxidant effect. potent inhibition of cancer cell proliferation. and cytotoxic activity. The most frequently employed method for preparing flavanones involves the oxidative cyclization of $2^{\prime}$-hydroxychalcones. " The treatment of $2^{\prime}$ hydroxychalcones with Co(salpr) in methanol under oxygen. ${ }^{3} \mathrm{~K}_{3} \mathrm{Fe}(\mathrm{CN})_{6}$ using phase transfer cataly'sis ${ }^{+}$and photochemical irradiation ${ }^{5}$ gives flavanones. but the yields are mostly' low to moderate. 2'-Hydroxychalcones are also converted to flavanones by the treatment with acidic reagents ${ }^{6}$ such as $\mathrm{H}_{2} \mathrm{SO}_{4}$ in methanol. ${ }^{6 \mathrm{a}} \mathrm{KF}$-celite in methanol. ${ }^{6 \mathrm{~b}}$ $\mathrm{CF}_{3} \mathrm{COOH}^{\infty}$ and polyphosphoric acid ${ }^{k d}$ or basic reagents ${ }^{7}$ such as pyridine. ${ }^{7 \mathrm{a}} \mathrm{KOH}$ in methanol. ${ }^{7 \mathrm{t}}$ and DBU in microwave irradiation. ${ }^{78}$ but most procedures are performed at high temperatures. Altematively flavanones are prepared by the cyclization of 3-bromo-1-phenylprop-2-ynyl aryl ethers in the presence of $\mathrm{Hg}\left(\mathrm{OCOCF}_{3}\right)_{2}$ after $\mathrm{NaBH}_{4}$ workup or the dehydration/cyclization of 3-hydroxy-1-o-hydroxypheny1-3-phenylpropan-1-ones derived from isoxazoles. but the former fails to furnish the methoxy substituted flavanones and the latter proceeds in four steps.

However there are no reports on the preparation of flavanones by the cyclodehydration of 1-(2'-hydroxyphenyl)-1-oxo-propan-3-phenyl-3-ols which are prepared from 2'-hydroxyacetophenones and benzaldehydes. This sy'nthetic strategy can avoid the undesirable reaction such as the partial cyclization of 2'-hydroxychalcones to flavanones during the synthesis. ${ }^{11}$ Although 1-(2'-hydroxyphenyl)-l-oxopropan-3-ols. prepared from 2'-hydroxyacetophenones and aldehydes or ketones with LDA. cyclize with acidic reagents ${ }^{11}$ such as $\mathrm{HCl}$ in methanol ${ }^{1 \mathrm{la}}$ and $\mathrm{H}_{2} \mathrm{SO}_{4}$ in $\mathrm{HOAc}^{1 \mathrm{ll}}$ or $\mathrm{HMPT}^{12}$ to give the corresponding chroman-4-ones. all these methods work only at high temiperatures and furthermore there are no reports on the preparation of flavanones with 2-substituted phenyl groups. In this paper we report that flavanones can be efficiently' synthesized via 1-(2'-hydroxyphenyl)-1-oxo-propan-3-phenyl-3-ols from the starting 2-hy'droxybenzoic acids in three steps.

2'-Hydroxyacetophenones 2 were readily prepared by the treatment of 2-hydroxybenzoic acids 1 with 3 equiv of $\mathrm{CH}_{3} \mathrm{Li}$ in THF in $74-93 \%$ yields according to our previous method. ${ }^{13}$ 1-(2'-Hy'droxyphenyl)-1-oxo-propan-3-phenyl-3ols 4 . precursors of flavanones 5 , were prepared by the treatment of the lithium dianions of 2 with benzaldehydes 3 (Scheme 1). To a yellowish solution of the lithium dianions. generated from 2 and 2 equiv of LDA in THF for $1.5 \mathrm{~h}$ at $-20^{\circ} \mathrm{C}$. were added 3 at $-78^{\circ} \mathrm{C}$. After being stirred for $1 \mathrm{~h}$ at $-78^{\circ} \mathrm{C}$. the mixture was quenched with sat. $\mathrm{NH}_{4} \mathrm{Cl}$ solution. The condensed residue after usual workup was purified by silica gel chromatography using $20 \% \mathrm{EtOAc} / n$-hexane to afford 4 in $81-94 \%$ yields without the formation of the corresponding 2'-hydroxychalcones 6 .

With 4 in hand. the cyclodelydration of 4 to 5 was studied<smiles>[R]c1cc(O)c(C(=O)O)c([R])c1[R]</smiles>

1

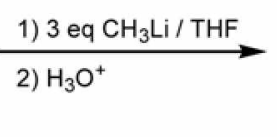<smiles>[R]c1cc(O)c(C(C)=O)c([R])c1[R]</smiles>

2<smiles>[R]c1ccc(C=O)cc1[R]</smiles>

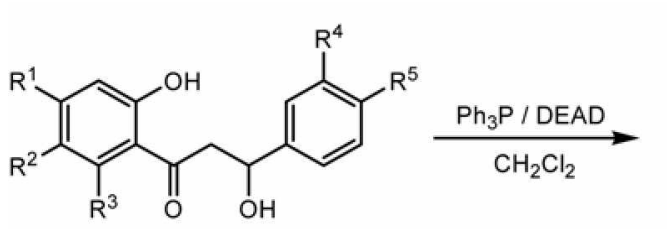<smiles>[R]c1ccc(C2CC(=O)c3c(cc([R])c([R])c3[R])O2)cc1[R1]</smiles>

3<smiles>[R7]c1ccc(/C=C/C(=O)c2c(O)cc([R])c([R])c2[R])cc1[R]</smiles>

6

$R^{1} \cdot R^{2} \cdot R^{3}=H, O C H_{3}: R^{4}, R^{5}=H, O C H_{3}, C l$ 
Table 1. Preparation of Flavanones from 2-Hydroxybenzoic Acids

\begin{tabular}{|c|c|c|c|c|c|c|}
\hline $\begin{array}{c}\text { Flavanones } \\
5\end{array}$ & $\mathrm{R}^{1}$ & $\mathrm{R}^{2}$ & $\mathrm{R}^{\vdots}$ & $\mathrm{R}^{4}$ & $\mathrm{R}^{\vdots}$ & $\begin{array}{l}\text { Isolated } \\
\text { yields, } \% \%^{a}\end{array}$ \\
\hline a & $\mathrm{H}$ & $\mathrm{H}$ & $\mathrm{H}$ & $\mathrm{H}$ & $\mathrm{H}$ & 64 \\
\hline b) & $\mathrm{H}$ & $\mathrm{H}$ & $\mathrm{H}$ & $\mathrm{H}$ & $\mathrm{Cl}$ & 60 \\
\hline c & $\mathrm{H}$ & $\mathrm{H}$ & $\mathrm{H}$ & $\mathrm{H}$ & $\mathrm{OCH}_{3}$ & 64 \\
\hline$d$ & $\mathrm{H}$ & $\mathrm{H}$ & $\mathrm{H}$ & $\mathrm{OCH}_{3}$ & $\mathrm{OCH}_{3}$ & 56 \\
\hline e & $\mathrm{OCH}_{3}$ & $\mathrm{H}$ & $\mathrm{H}$ & $\mathrm{H}$ & $\mathrm{H}$ & 63 \\
\hline$f$ & $\mathrm{OCH}_{3}$ & $\mathrm{H}$ & $\mathrm{H}$ & $\mathrm{H}$ & $\mathrm{Cl}$ & 70 \\
\hline g & $\mathrm{OCH}_{3}$ & $\mathrm{H}$ & $\mathrm{H}$ & $\mathrm{H}$ & $\mathrm{OCH}_{3}$ & 68 \\
\hline $\mathrm{h}$ & $\mathrm{H}$ & $\mathrm{OCH}_{3}$ & $\mathrm{H}$ & $\mathrm{H}$ & $\mathrm{H}$ & 69 \\
\hline i & $\mathrm{H}$ & $\mathrm{OCH}_{3}$ & $\mathrm{H}$ & $\mathrm{H}$ & $\mathrm{Cl}$ & 59 \\
\hline $\mathbf{j}$ & $\mathrm{H}$ & $\mathrm{OCH}_{3}$ & $\mathrm{H}$ & $\mathrm{H}$ & $\mathrm{OCH}_{3}$ & 61 \\
\hline$k$ & $\mathrm{H}$ & $\mathrm{H}$ & $\mathrm{OCH}_{3}$ & $\mathrm{H}$ & $\mathrm{H}$ & 51 \\
\hline I & $\mathrm{H}$ & $\mathrm{H}$ & $\mathrm{OCH}_{3}$ & $\mathrm{H}$ & $\mathrm{OCH}_{3}$ & 55 \\
\hline
\end{tabular}

"Overall yields of three steps from the starting 2-hydroxybenzoic acids.

using l-(2'-hydroxyphenyl)-1-oxo-propan-3-(4'-methoxyphenyl)-3-ol $t c$ as a model substrate. Initially the cyclodehydration was attempted by treating $+c$ with $\mathrm{Ph}_{3} \mathrm{P} / \mathrm{CCl}_{4} /$ $\mathrm{Et}_{3} \mathrm{~N}^{14}$ in $\mathrm{CH}_{3} \mathrm{CN}$ or $\mathrm{DMF} /(\mathrm{COCl})_{2} / \mathrm{Et}_{3} \mathrm{~N}$ (Vilsmeier reagent) ${ }^{15}$ in $\mathrm{CH}_{2} \mathrm{Cl}_{2}$. Under these reaction conditions. to our disappointment. desired 4 '-methoxyflavanone $\mathbf{5} \mathbf{c}$ was obtained as a minor product with chalcone $\mathbf{6 c}$ as a major product in $82 \%$ and $80 \%$ yield. respectively, in a ratio of $1: 4$ and $1: 3$. respectively. detected by ${ }^{1} \mathrm{H}$ NMR integration. The cyclodehydration of tc was next examined with $\mathrm{Ph}_{3} \mathrm{P} /$ diethyl azodicarboxylate (DEAD) ${ }^{16}$ varying solvents. The treatment of $4 \mathbf{c}$ with $\mathrm{Ph}_{3} \mathrm{P} / \mathrm{DEAD}$ in $\mathrm{CH}_{2} \mathrm{Cl}_{2}$. THF, $\mathrm{CH}_{3} \mathrm{CN}$ at $0{ }^{\circ} \mathrm{C}$ gave $\overline{\mathbf{s}}$ and $6 \mathrm{c}$ with a ratio of $13: 1.6: 1$, and $1.5: 1$, respectively. in $99 \% .98 \%$. and $94 \%$ yield. respectively. Thus. the cyclodehydration of 4 was carried out by the slow addition of DEAD to a mixture solution of 4 and $\mathrm{Ph}_{3} \mathrm{P}$ in $\mathrm{CH}_{2} \mathrm{Cl}_{2}$ at 0 ${ }^{\circ} \mathrm{C}$. After completion of the reaction, the condensed residue was subjected to a short pathway silica gel chromatography using $30 \% \mathrm{EtOAc} / n$-hexane to eliminate $\mathrm{Ph}_{\hat{j}} \mathrm{P}=\mathrm{O}$ and diethyl 1,2-hydrazinedicarboxylate. The successive recrystallization of the condensed mixture in $n$-hexane gave pure 5 in $76-89 \%$ yields.

As shown in Table 1. various flavanones were synthesized in high overall yields $(51-70 \%)$ from the starting 1 . The reaction worked well both for the methoxy substituent (5e5j) on the A-ring and the chloro (5b. 5f. 5i) or methoxy substituent $(5 \mathrm{c} .5 \mathrm{~d} .5 \mathrm{~g} .5 \mathrm{j})$ on the B-ring of 5 regardless of the kind and the position of substituents under the present reaction conditions. Furthermore, the ortho substituted methoxy group (5k. 5) of A-ring didn't influence the cyclodehydration of 4 . Thus. the present method provides (i) a novel synthesis of 5 from $\beta$-hydroxy ketone intermediates 4 (ii) the avoidance of isomerization between 2'-lydroxychalcones and flavanones (iii) the high rield of 5 .

\section{Experimental Section}

Preparation of 1-(2'-hydroxyphenyl)-1-oxo-propan-3phenyl-3-ol ta (General procedure). To a solution of 2'- hydroxyacetophenone $(545 \mathrm{mg} .4 .0 \mathrm{mmol})$ in THF (12 mL) was added lithium dissopropylamide (2.0 M. $4.2 \mathrm{~mL}, 8.4$ numol) at $-20^{\circ} \mathrm{C}$ under argon atmosphere and stirred for 1.5 $\mathrm{h}$. The mixture was then cooled to $-78^{\circ} \mathrm{C}$ and a solution of benzaldelyde $(425 \mathrm{mg} .4 .0 \mathrm{mmol})$ in THF $(8 \mathrm{~mL})$ was added. After being stirred for $\mathrm{l} h$ at $-78^{\circ} \mathrm{C}$. the mixture was quenched with sat. $\mathrm{NH}_{4} \mathrm{Cl}(3 \mathrm{~mL})$. After evaporation of THF. the mixture was poured into sat. $\mathrm{NH}_{4} \mathrm{Cl}(30 \mathrm{~mL})$ and the aqueous phase was extracted with methylene chloride ( 3 $\times 25 \mathrm{~mL}$ ). The combined organic phases were dried over $\mathrm{MgSO}_{4}$. filtered, and concentrated in vacho. The residue was purified by silica gel column chromatography using $20 \%$ EtOAc/n-hexane to give ta $(824 \mathrm{mg}, 85 \%)$. M.p. $75-76^{\circ} \mathrm{C}$ : ${ }^{1} \mathrm{H}$ NMR $\left(300 \mathrm{MHz}, \mathrm{CDCl}_{3}\right.$ ) $\delta 12.08$ (s. $\left.1 \mathrm{H}\right), 7.70$ (dd, $J_{1}=$ $\left.8.1 \mathrm{~Hz} . J_{2}=1.6 \mathrm{~Hz} . \mathrm{lH}\right) .7 .28-7.52(\mathrm{~m}, 6 \mathrm{H}), 7.00\left(\mathrm{dd}, J_{1}=\right.$ $\left.8.4 \mathrm{~Hz} . J_{2}=0.9 \mathrm{~Hz} . \mathrm{lH}\right) .6 .85 .6 .91(\mathrm{~m}, \mathrm{lH}), 5.37\left(\mathrm{dd}, J_{1}=\right.$ $\left.8.8 \mathrm{~Hz} . J_{2}=3.3 \mathrm{~Hz} .1 \mathrm{H}\right), 3.46\left(\mathrm{dd}, J_{1}=17.6 \mathrm{~Hz} . J_{2}=8.8 \mathrm{~Hz}\right.$, lH). $3.36\left(\mathrm{dd}, J_{1}=17.6 \mathrm{~Hz} . J_{2}=3.3 \mathrm{~Hz} . \mathrm{lH}\right) .3 .22(\mathrm{br} \mathrm{s}, \mathrm{lH})$ : ${ }^{13} \mathrm{C}$ NMR $\left(75 \mathrm{MHz} . \mathrm{CDCl}_{3}\right) \delta 205.9 .163 .0 .143 .1,137.3$. $130.5,129.1,128.3,126.1,119.8 .119 .5 .119 .1,70.3 .47 .6$ : FT-IR (KBr) $3439(\mathrm{O}-\mathrm{H}), 3032.2907,1637(\mathrm{C}=\mathrm{O}), 1579$, 1448. $1268,1061,754,701 \mathrm{~cm}^{-1}$ : Ms mz (\%) $224\left(\mathrm{M}^{-}-18\right.$, 82). $223(81) .147(57) .120(100), 104(43), 92(57) .77$ (17).

Preparation of flavanone $5 \mathbf{a}$ (General procedure). To a mixture solution of $4 a(727 \mathrm{mg} .3 .0 \mathrm{mmol})$ and triphenylphosphine ( $787 \mathrm{mg} .3 .0 \mathrm{mmol}$ ) in methylene chloride (18 $\mathrm{mL}$ ) was slowly added diethyl azodicarboxylate (40 wt \% in toluene, $1.5 \mathrm{~mL} .3 .3 \mathrm{mmol}$ ) at $0{ }^{\circ} \mathrm{C}$ over $3 \mathrm{~min}$. After being stirred for $10 \mathrm{~min}$. the solvent of the resulting yellow mixture was evaporated in vacto. The residue was subjected to a short pathway silica gel colunn clromatography using $30 \%$ EtOAc/n-hexane to give a mixture of flavanone and the corresponding chalcone in a ratio of $13: 1(639 \mathrm{mg} .95 \%)$. The mixture was recrystallized in $n$-hexane to give $\mathbf{5 a}(579$ mg. $86 \%$ ) as a colorless solid. M.p. $74-75^{\circ} \mathrm{C}$ (lit. ${ }^{4} 76{ }^{\circ} \mathrm{C}$ ), ${ }^{1} \mathrm{H}$ $\operatorname{NMR}\left(300 \mathrm{MHz} . \mathrm{CDCl}_{3}\right) \delta 7.94\left(\mathrm{dd}, J_{1}=8.1 \mathrm{~Hz} . J_{2}=1.7\right.$ Hz. $1 \mathrm{H}$ ). $7.36-7.55$ (m. $6 \mathrm{H}$ ). $7.04-7.09$ (m. $2 \mathrm{H}$ ). 5.49 (dd. $J_{1}$ $\left.=13.2 \mathrm{~Hz}, J_{2}=3.0 \mathrm{~Hz} .1 \mathrm{H}\right) .3 .10\left(\mathrm{dd} . J_{1}=16.9 \mathrm{~Hz}, J_{2}=13.2\right.$ Hz. $1 \mathrm{H}) .2 .90\left(\mathrm{dd} . J_{1}=16.9 \mathrm{~Hz}, J_{2}=3.0 \mathrm{~Hz}, 1 \mathrm{H}\right) ; \mathrm{FT}-\mathrm{IR}$ (KBr) $3035,2962.1688(\mathrm{C}=\mathrm{O}), 1606,1462,1304,1228$. 1065. $760.696 \mathrm{~cm}^{-1} .{ }^{13} \mathrm{C}$ NMR $\left(75 \mathrm{MHz} . \mathrm{CDCl}_{3}\right) \delta 192.4$. 162.0. 139.1, 136.6. 129.3, 129.2. 127.5, 126.6. 122.0, 121.3. $118.5,80.0 .45 .1$ : Ms $m z(\%) 224\left(\mathrm{M}^{+} .100\right), 223(93) .147$ (54). $120(83) .92(44), 77$ (11).

4'-Chloroflavanone (5b). M.p. $84-85^{\circ} \mathrm{C}\left(\right.$ lit. $\left.^{17} 85^{\circ} \mathrm{C}\right),{ }^{1} \mathrm{H}$ $\operatorname{NMR}\left(300 \mathrm{MHz} . \mathrm{CDCl}_{3}\right) \delta 7.93\left(\mathrm{dd}, J_{1}=7.8 \mathrm{~Hz} . J_{2}=1.6\right.$ Hz. lH). 7.49-7.55 (m, lH). 7.39-7.45 (m. 4H). 7.03-7.10 (m. $2 \mathrm{H}) .5 .47$ (dd. $\left.J_{1}=13.0 \mathrm{~Hz} . J_{2}=3.2 \mathrm{~Hz}, \mathrm{lH}\right), 3.04(\mathrm{dd}$, $\left.J_{1}=16.8 \mathrm{~Hz}, J_{2}=13.0 \mathrm{~Hz}, 1 \mathrm{H}\right), 2.88\left(\mathrm{dd}, J_{1}=16.8 \mathrm{~Hz} . J_{2}=\right.$ $3.2 \mathrm{~Hz} . \mathrm{lH}):{ }^{13} \mathrm{C}$ NMR $\left(75 \mathrm{MHz}, \mathrm{CDCl}_{3}\right) \delta 191.5,161.3$, 137.3. 136.3, 134.6. 129.1. 127.5, 127.1. 121.8, 120.9. 118.1, 78.8. 44.6: FT-IR (KBr) 3036. 2977, $1697(\mathrm{C}=\mathrm{O}) .1602$. $1471.1152 .821 \mathrm{~cm}^{-1}$; Ms $m z(\%) 260\left(\mathrm{M}^{-}+2.33\right), 259(42)$, $258\left(\mathrm{M}^{+} .100\right) .257(85) .147(44) .120(90), 92(33)$.

4'-Methoxyflavanone (5c). M.p. $96-97^{\circ} \mathrm{C}$ (lit. $\left.{ }^{+} 98^{\circ} \mathrm{C}\right) ;{ }^{\circ} \mathrm{H}$ NMR $\left(300 \mathrm{MHz} . \mathrm{CDCl}_{3}\right) \delta 7.93$ (dd, $J_{1}=8.3 \mathrm{~Hz} . J_{2}=1.7$ 
Hz, IH). $7.46-7.53$ (m. IH). 7.41 (d. $J=8.7$ Hz. $2 \mathrm{H}$ ), 7.02 $7.07(\mathrm{~m}, 2 \mathrm{H}), 6.96(\mathrm{~d} . J=8.7 \mathrm{~Hz} .2 \mathrm{H}) .5 .43\left(\mathrm{dd} . J_{1}=13.3\right.$ $\left.\mathrm{Hz}, J_{2}=2.8 \mathrm{~Hz} . \mathrm{lH}\right) .3 .83$ (s. $3 \mathrm{H}$ ). $3.1 \mathrm{l}$ (dd. $J_{1}=16.8 \mathrm{~Hz} . J_{2}$ $=13.3 \mathrm{~Hz}, 1 \mathrm{H}), 2.86\left(\mathrm{dd}, J_{1}=16.8 \mathrm{~Hz} . J_{2}=2.8 \mathrm{~Hz}, 1 \mathrm{H}\right) ;{ }^{13} \mathrm{C}$ NMR $\left(75 \mathrm{MHz}^{\left.-\mathrm{CDCl}_{3}\right)} \delta\right.$ 192.7. 162.0. 160.4. 136.6. 131.2. 128.1. 127.4. 121.9, 121.3. 118.5. 114.6, 79.8, 55.8, 44.9: FT-IR (KBr) 3007, 2963, $1690(\mathrm{C}=0), 1614,1463.1252$. 1028. $826 \mathrm{~cm}^{-1}$ : Ms $m z(\%) 254\left(\mathrm{M}^{+}, 64\right) .253(48) .147$ (16), $134(100) .121(27) .92(10)$.

3',4'-Dimethoxyflavanone (5d). M.p. $123-124{ }^{\circ} \mathrm{C} ;{ }^{1} \mathrm{H}$ NMR $\left(300 \mathrm{MHz}, \mathrm{CDCl}_{3}\right) \delta 7.93$ (dd. $J_{1}=7.9 \mathrm{~Hz} . J_{2}=1.5$ Hz, IH), 7.46-7.53 (m. IH), 6.97-7.07 (m, $4 \mathrm{H}) .6 .90(\mathrm{~d}, J=$ $7.9 \mathrm{~Hz} . \mathrm{lH}) .5 .42\left(\mathrm{dd}, J_{1}=13.2 \mathrm{~Hz}, J_{2}=2.7 \mathrm{~Hz}, 1 \mathrm{H}\right), 3.92(\mathrm{~s}$. $3 \mathrm{H}) .3 .90$ (s. $3 \mathrm{H}) .3 .11\left(\mathrm{dd}, J_{1}=16.8 \mathrm{~Hz}, J_{2}=13.2 \mathrm{~Hz} . \mathrm{HH}\right)$. 2.87 (dd. $\left.J_{1}=16.8 \mathrm{~Hz}, J_{2}=2.8 \mathrm{~Hz} .1 \mathrm{H}\right) \cdot{ }^{13} \mathrm{C} \mathrm{NMR}(75 \mathrm{MHz}$. $\left.\mathrm{CDCl}_{3}\right) \delta$ 192.2. 161.6. 149.4, 149.3, 136.2, 131.2. 127.1. 121.6. 120.9. 118.8, 118.2, 111.1. 109.4, 79.6. 56.0 (overlapped $\mathrm{OCH}_{3}$ ). 44.6: FT-IR (KBr) 3002, 2936. $1687(\mathrm{C}=\mathrm{O})$. 1607. 1464. 1266. $11+1,1027,763 \mathrm{~cm}^{-1}$ : Ms $m z$ (\%) 284 $\left(\mathrm{M}^{+}, 100\right), 283(39), 253(17), 164(86), 15 \mathrm{l}(47), 12 \mathrm{l}(20)$.

7-Methoxyflavanone (5e). M.p. $89-90{ }^{\circ} \mathrm{C}$ (lit. ${ }^{18} 90-91$ $\left.{ }^{\circ} \mathrm{C}\right):{ }^{1} \mathrm{H}$ NMR $\left(300 \mathrm{MHz} . \mathrm{CDCl}_{3}\right) \delta 7.88(\mathrm{~d} . J=8.8 \mathrm{~Hz} . \mathrm{lH})$. $7.38-7.50(\mathrm{~m}, 5 \mathrm{H}), 6.62\left(\mathrm{dd}, J_{1}=8.8 \mathrm{~Hz} . J_{2}=2.4 \mathrm{~Hz} . \mathrm{lH}\right.$ ). $6.51(\mathrm{~d} . J=2.4 \mathrm{~Hz} .2 \mathrm{H}) .5 .47\left(\mathrm{dd}, J_{1}=13.2 \mathrm{~Hz} . J_{2}=3.0 \mathrm{~Hz}\right.$. $1 \mathrm{H}), 3.84$ (s. $3 \mathrm{H}) .3 .05$ (dd. $J_{1}=16.9 \mathrm{~Hz}, J_{2}=13.2 \mathrm{~Hz} . \mathrm{HH}$ ). $2.83\left(\right.$ dd. $\left.J_{1}=16.9 \mathrm{~Hz}, J_{2}=3.1 \mathrm{~Hz} .1 \mathrm{H}\right) \cdot{ }^{13} \mathrm{C} \mathrm{NMR}(75 \mathrm{MHz}$. $\left.\mathrm{CDCl}_{3}\right) \delta 191.0,166.6 .163 .9 .139 .2 .129 .3,129.2,126.6$ (overlapped). 115.2. 110.7. 101.3, 80.4, 56.1. 44.7: FT-IR $(\mathrm{KBr}) 3014,2970.1679(\mathrm{C}=\mathrm{O}) .1610,1442,1258,1156$. $746,700 \mathrm{~cm}^{-1}$ : Ms $m \mathrm{z}(\%) 254\left(\mathrm{M}^{+} .100\right) .253(65) .177$ (63), $150(65), 122(32), 77(10)$.

4'-Chloro-7-methoxyflavanone (5f). M.p. $120-122{ }^{\circ} \mathrm{C}$ (lit. $\left.{ }^{17} 120-121{ }^{\circ} \mathrm{C}\right):{ }^{1} \mathrm{H}$ NMR $\left(300 \mathrm{MHz} . \mathrm{CDCl}_{3}\right) \delta 7.87\left(\mathrm{~d}_{,} J\right.$ $=8.8 \mathrm{~Hz}, \mathrm{lH}) .7 .38-7.45(\mathrm{~m} .4 \mathrm{H}) .6 .63\left(\mathrm{dd} . J_{1}=8.8 \mathrm{~Hz} . J_{\Sigma}=\right.$ $2.4 \mathrm{~Hz}, \mathrm{IH}) .6 .49(\mathrm{~d}, J=2.4 \mathrm{~Hz}, \mathrm{IH}) .5 .45\left(\mathrm{dd}, J_{1}=12.9 \mathrm{~Hz}\right.$. $J_{\Sigma}=3.2 \mathrm{~Hz}, 1 \mathrm{H}$ ), 3.84 (s. $3 \mathrm{H}$ ). 2.99 (dd. $J_{1}=16.8 \mathrm{~Hz} . J_{2}=$ $12.9 \mathrm{~Hz} . \mathrm{IH}$ ). $2.82\left(\mathrm{dd}, J_{1}=16.8 \mathrm{~Hz} \cdot J_{2}=3.2 \mathrm{~Hz} \cdot \mathrm{IH}\right.$ ): ${ }^{13} \mathrm{C}$ $\operatorname{NMR}\left(75 \mathrm{MHz}^{\left.-\mathrm{CDCl}_{\hat{j}}\right)} \delta\right.$ 190.5. 166.6. 163.7. 137.7. 135.0. 129.4. 129.2. 127.9, 115.2. 110.8, 101.3. 79.6, 56.1, 44.6: FT-IR $(\mathrm{KBr}) 3021,2967,1682(\mathrm{C}=\mathrm{O}), 1609,1493,1259$. 1092. $828 \mathrm{~cm}^{-1}$ : Ms mz (\%) $290\left(\mathrm{M}^{+}+2.33\right) .289(40) .288$ $\left(\mathrm{M}^{+}, 100\right), 287(64), 177(63), 150(84), 122(42), 107(22)$.

4',7-Dimethoxyflavanone (5g). M.p. $93-94{ }^{\circ} \mathrm{C}$ (lit. ${ }^{18} 94-$ $\left.95^{\circ} \mathrm{C}\right) ;{ }^{1} \mathrm{H} \mathrm{NMR}\left(300 \mathrm{MHz}, \mathrm{CDCl}_{3}\right) \delta 7.87(\mathrm{~d} . J=8.8 \mathrm{~Hz}$. $1 \mathrm{H}), 7.40(\mathrm{~d}, J=6.7 \mathrm{~Hz} .2 \mathrm{H}) .6 .96(\mathrm{~d}, J=6.7 \mathrm{~Hz} .2 \mathrm{H}) .6 .61$ (dd. $\left.J_{1}=8.8 \mathrm{~Hz} . J_{2}=2.4 \mathrm{~Hz}, 1 \mathrm{H}\right) .6 .48($ d. $J=2.4 \mathrm{~Hz}, 1 \mathrm{H})$. 5.42 (dd. $J_{1}=13.2 \mathrm{~Hz}, J_{2}=2.9 \mathrm{~Hz}, \mathrm{IH}$ ), 3.83 (overlapped OCH 3 s. $6 \mathrm{H}$ ). 3.06 (dd. $J_{1}=16.9 \mathrm{~Hz}, J_{2}=13.2 \mathrm{~Hz} . \mathrm{lH}$ ). 2.80 $\left(\right.$ dd. $\left.J_{1}=16.9 \mathrm{~Hz} . J_{\hat{乏}}=2.9 \mathrm{~Hz} .1 \mathrm{H}\right):{ }^{13} \mathrm{C}$ NMR $(75 \mathrm{MHz}$. $\left.\mathrm{CDCl}_{3}\right) \delta$ 191.2. 166.6. 164.0, 160.4, 131.2, 129.1. 128.1. 115.2. 114.6. 110.6, 101.3. 80.2. 56.0, 55.8. 44.5; FT-IR $(\mathrm{KBr}) 3005,2963,1679(\mathrm{C}=\mathrm{O}), 1610,1444,1257,1159$. 1027. $836 \mathrm{~cm}^{-1}$; Ms $m z(\%) 284\left(\mathrm{M}^{-}, 100\right) .283(57), 177$ (20), 134 (99), $121(40), 91(18)$.

6-Methoxyflavanone (5h). M.p. $142-143^{\circ} \mathrm{C}$ (lit. ${ }^{18} 140$ $1+2{ }^{\circ} \mathrm{C}$ ): ${ }^{1} \mathrm{H}$ NMR $\left(300 \mathrm{MHz} . \mathrm{CDCl}_{3}\right) \delta 7.37-7.50$ (m. $5 \mathrm{H}$ ). $7.36(\mathrm{~d}, J=3.1 \mathrm{~Hz}, 1 \mathrm{H}) .7 .13\left(\mathrm{dd} . J_{1}=9.0 \mathrm{~Hz}, J_{2}=3.1 \mathrm{~Hz}\right.$.
$1 \mathrm{H}$ ). $6.99(\mathrm{~d} . J=9.0 \mathrm{~Hz} .1 \mathrm{H}), 5.44\left(\mathrm{dd} . J_{1}=13.3 \mathrm{~Hz}, J_{2}=3.0\right.$ Hz. $1 \mathrm{H}), 3.82$ (s. $3 \mathrm{H}), 3.07\left(\mathrm{dd}, J_{1}=17.0 \mathrm{~Hz}, J_{2}=13.3 \mathrm{~Hz}\right.$, lH). $2.88\left(\mathrm{dd} . J_{1}=17.0 \mathrm{~Hz}, J_{2}=3.0 \mathrm{~Hz} .1 \mathrm{H}\right) ;{ }^{13} \mathrm{C} \operatorname{NMR}(75$ $\left.\mathrm{MHz}_{3} \mathrm{CDCl}_{3}\right) \delta 192.5,156.7 .154 .6,139.2 .129 .2,129.1$, 126.5. 125.8. 121.2. 119.8, 107.7. 80.1. 56.2. 45.0, FT-IR (KBr) 3010, 2960. 1674 (C=O). 1616, 1461. 1226, 1034. $769.696 \mathrm{~cm}^{-1}$. Ms $m z(\%) 254\left(\mathrm{M}^{-}, 29\right) .253(18) .177(16)$, $150(100), 107(18)$.

4'Chloro-6-methoxyflavanone (5i). M.p. 114-115 $\mathrm{C}$ (lit. ${ }^{17} 115-116^{\circ} \mathrm{C}$ ): ${ }^{1} \mathrm{H}$ NMR $\left(300 \mathrm{MHz}, \mathrm{CDCl}_{3}\right.$ ) $\delta 7.38-7.44$ $(\mathrm{m}, 4 \mathrm{H}), 7.35(\mathrm{~d}, J=3.2 \mathrm{~Hz}, \mathrm{lH}), 7.13\left(\mathrm{dd} . J_{1}=9.0 \mathrm{~Hz} . J_{2}=\right.$ $3.2 \mathrm{~Hz}, \mathrm{lH}) .6 .98(\mathrm{~d}, J=9.0 \mathrm{~Hz}, \mathrm{lH}) .5 .42\left(\mathrm{dd} . J_{1}=13.0 \mathrm{~Hz}\right.$, $J_{2}=3.2 \mathrm{~Hz} .1 \mathrm{H}$ ). $3.82(\mathrm{~s} .3 \mathrm{H}) .3 .02\left(\mathrm{dd} . J_{1}=16.9 \mathrm{~Hz}, J_{2}=\right.$ $13.0 \mathrm{~Hz}, 1 \mathrm{H}) .2 .86\left(\right.$ dd. $\left.J_{1}=16.9 \mathrm{~Hz}, J_{2}=3.2 \mathrm{~Hz} .1 \mathrm{H}\right) ;{ }^{12} \mathrm{C}$ $\operatorname{NMR}\left(75 \mathrm{MHz}, \mathrm{CDCl}_{3}\right) \delta 192.0,156.4,154.7,137.8,134.9$, $129.4,127.9,125.9,121.1,119.8 .107 .8,79.3 .56 .2 .44 .9$ : FT-IR (KBr) 3013, 2951, I686 (C=0). 1615. 1489. 1280. 1034. $825.689 \mathrm{~cm}^{-1} ; \mathrm{Ms} m z(\%) 290\left(\mathrm{M}^{+}+2,19\right), 288\left(\mathrm{M}^{-}\right.$. $57) .177(9), 150(100) .135(8), 107(17)$.

4',6-Dimethoxyflavanone (5j). M.p. $158-159{ }^{\circ} \mathrm{C}$ (lit. ${ }^{18}$ $159-160^{\circ} \mathrm{C}$ ); ${ }^{1} \mathrm{H}$ NMR $\left(300 \mathrm{MHz} . \mathrm{CDCl}_{3}\right) \delta 7.41$ (d. $J=8.8$ Hz. $2 \mathrm{H}), 7.35(\mathrm{~d}, J=3.2 \mathrm{~Hz} .1 \mathrm{H}), 7.11$ (dd. $J_{1}=9.0 \mathrm{~Hz} . J_{2}=$ $3.2 \mathrm{~Hz}, 1 \mathrm{H}), 6.98(\mathrm{~d} . J=9.0 \mathrm{~Hz}, \mathrm{IH}), 6.96(\mathrm{~d} . J=8.8 \mathrm{~Hz}$. $2 \mathrm{H}) .5 .38\left(\mathrm{dd}, J_{1}=13.3 \mathrm{~Hz} . J_{2}=2.9 \mathrm{~Hz} .1 \mathrm{H}\right) .3 .83(\mathrm{~s}, 3 \mathrm{H})$, 3.82 (s. $3 \mathrm{H}$ ). 3.08 (d. $J_{1}=16.9 \mathrm{~Hz} . J_{2}=13.3 \mathrm{~Hz} .1 \mathrm{H}$ ). 2.84 (dd. $\left.J_{1}=16.9 \mathrm{~Hz}, J_{2}=2.9 \mathrm{~Hz}, 1 \mathrm{H}\right) ;{ }^{12} \mathrm{C}$ NMR $(75 \mathrm{MHz}$, $\left.\mathrm{CDCl}_{3}\right) \delta 192.3,159.9,156.4,154.2 .130 .9$. 127.7. 125.4. 120.7, 119.4. 114.2. 107.3. 79.5, 55.8. 55.4. 44.4: FT-IR $(\mathrm{KBr}) 3007,2970,1679(\mathrm{C}=\mathrm{O}) .1613,1487,1250,1029$. $830 \mathrm{~cm}^{-1}$; Ms $m z(\%) 284\left(\mathrm{M}^{+} .100\right) .283(18), 150(81)$, $134(91), 107(13)$.

5-Methoxyflavanone (5k). M.p. $146-147{ }^{\circ} \mathrm{C}:{ }^{~} \mathrm{H}$ NMR $\left(300 \mathrm{MHz} . \mathrm{CDCl}_{3}\right) \delta 7.37-7.46(\mathrm{~m} .6 \mathrm{H}) .6 .66(\mathrm{~d} . J=8.4 \mathrm{~Hz}$, lH). $6.55\left(\right.$ d. $J=8.4 \mathrm{~Hz} .1 \mathrm{H}$ ), 5.43 (dd. $J_{1}=13.1 \mathrm{~Hz}, J_{2}=3.0$ Hz. $\mathrm{lH}$ ), 3.93 (s. $3 \mathrm{H}$ ), 3.07 (dd, $J_{1}=16.4 \mathrm{~Hz}, J_{2}=13.1 \mathrm{~Hz}$, $1 \mathrm{H}) .2 .85\left(\mathrm{dd} . J_{1}=16.4 \mathrm{~Hz}, J_{2}=3.0 \mathrm{~Hz} \cdot 1 \mathrm{H}\right) \cdot{ }^{13} \mathrm{C}$ NMR $(75$ $\left.\mathrm{MHz}, \mathrm{CDCl}_{3}\right) \delta 190.7,163.2 .160 .8,138.7 .136 .0,128.8$, 128.7. 126.1. 111.4. 110.2. 104.0. 78.9, 56.2, 46.0: FT-IR $(\mathrm{KBr}) 3020,2976,1675(\mathrm{C}=\mathrm{O}), 1599,1460,1252,1089$. $750.691 \mathrm{~cm}^{-1}: \mathrm{Ms} m z(\%) 254\left(\mathrm{M}^{-}, 57\right) .253(44) .177(30)$. $150(100), 122(31), 107(39)$.

4',5-Dimethoxyflavanone (51). M.p. $104-105{ }^{\circ} \mathrm{C} ;{ }^{1} \mathrm{H}$ NMR (300 MHz. $\left.\mathrm{CDCl}_{2}\right) \delta 7.37-7.42(\mathrm{~m} .3 \mathrm{H}) .6 .95(\mathrm{~d}, J=$ $8.8 \mathrm{~Hz}, 2 \mathrm{H}), 6.64$ (d. $J=8.3 \mathrm{~Hz}, \mathrm{lH}), 6.54$ (d. $J=8.3 \mathrm{~Hz}$, $1 \mathrm{H}) .5 .38$ (dd. $J_{1}=13.1 \mathrm{~Hz} . J_{2}=2.8 \mathrm{~Hz} .1 \mathrm{H}$ ). $3.93(\mathrm{~s}, 3 \mathrm{H})$, $3.83(\mathrm{~s}, 3 \mathrm{H}), 3.09\left(\mathrm{dd}, J_{1}=16.4 \mathrm{~Hz}, J_{2}=13.1 \mathrm{~Hz}, 1 \mathrm{H}\right), 2.82$ (dd. $\left.J_{1}=16.4 \mathrm{~Hz}, J_{2}=2.9 \mathrm{~Hz}, 1 \mathrm{H}\right) ;{ }^{13} \mathrm{C}$ NMR $(75 \mathrm{MHz}$, $\left.\mathrm{CDCl}_{3}\right) \delta 191.0,163.3,160.8,159.9 .136 .0 .130 .7 .127 .7$. $114.1,111.3 .110 .2$. 104.0. 78.7. 56.2, 55.4. 45.8: FT-IR (KBr) 3065, 2939. $1672(\mathrm{C}=0) .1603,1468,1253,1027$. $828 \mathrm{~cm}^{-1}:$ Ms $m z(\%) 284\left(\mathrm{M}^{+} .51\right) .283(52), 150(28) .134$ (100), $121(20), 107(21)$.

Acknowledgment. This work was supported by the Korea Research Foundation Grant funded by the Korea Govenment (MOEHRD). Basic Research Promotion Fund (KRF2005-005-J13001) 


\section{References}

1. (a) Harbone. J. B. The Flavonoids: Chapman \& Hall: London. U. K.. 1994 p 406. (b) Harbone, I. B.: Willians. C. A. Nat. Prod. Rep. 2001, 18, 310. (c) Nijveldt. R. I.: Nood, E.: Hoorn. D.: Boelens, P. G.; Norren. K.: Leelwen. P. Am. J. Chm. Aur: 2001. 74. 418. (d) Heim. K. E.: Tagliaferro. A. R.: Bobilya. D. J. J. Nutr. Biochem. 2002. 13. 572 .

2. (a) Bohm. B. A. Introduction to Flawonoids: Harwood Academic Publishers: Annsterdam. Netherlands. 1998: p 243. (b) Harborne, I. B. The Flaronoids: Chapman \& Hall: London, U. K. 1994: p 427.

3. Maruyama. K.: Tamanaka. K: Nishinaga, A.: Inada. A: Nakanishi. T. Tetrahedron Lett. 1989. 30.4145.

4. Makrandi. J. K.: Bala. S. Sinth. Comm. 2000. 30. 3555

5. Matsushima, R: Kagevama, H. J. Chem. Soc. Perhin Trons. II 1985,743 .

6. (a) Bidhendi. G. N.: Bannerjee, N. R. Indian J. Chem. 1989, 28 B. 352. (b) Harwood. L. M.: Lottus. G. C.: Oxford. A.: Thomson. C. Symth. Comm 1990. 20. 649. (c) Chaturvedi. R.: Patil. P. N.: Mulchandani. N. B. Indian J. Chent 1992.31B.340. (d) Paquette. L. A. Enruclopedia of Reagents for Organic Sumthesis: John Wiley \& Sons: New York. U. S. A.. 1995: p 4172 2.

7. (a) Dutta, C. P. Roy, L. P. K. Indiam J. Chem. 1975. 13. 425. (b)
Moorthy. N. S. H. N.: Singh. R. T.: Singh. H. P.: Gupta. S. D Chent. Pham Bull 2006. 5+. 1384. (c) Patonay. T: Varma. R. S.: Vass. A.: Levai. A.: Dudas. T. Tetrahedron Letl. 2001. +2.1403.

8. Subramanian, R. S.: Balasubramanian, K. K. J. Chem. Soc. Chem. Comm 1990, 1469

9. Thomsen. I.; Torssell. K. B. G. Acta Chem. Scand. Ser: B 1988. +2. 303 .

10. Hoshino. Y.: Takeno. N. Bull. Chem. Soc. Jpm. 1986.59.2903

11. (a) Banerij. A.: Goomer. N. C.: Kalena. G. P. Swh. Conm 1980. 10. 851. (b) Kelly. S. E.: Vanderplas, B. C. J. Org. Chem 1991. 56.1325 .

12. Banerji, A.: Kalena. G. P. Synth. Comm. 1989, 19. 159

13. Lee. J. I.: Son. H. S.: Park. H. Bull. Korean Chem. Soc. 2004. 25. 1945.

14. Einhom. I.: Einhom. C.: Luche. J. L. Swhth. Conm 1990. 20. 1105.

15. Procopiou, P. A.; Brodie. A. C.: Deal, M. J.; Hayman. D. F. Tetrahedron Lett. 1993. 34. 7483.

16. (a) Carlock. J. T.: Mack. M. P. Tetrahedron Lett. 1978. 19. 5153. (b) Subramanian. R. S.: Balasubramanian. K. K. Symh. Conmt. 1989. 19. 1255

17. Lee. J. I.; Jung. M. G. Bull. Korean Chem. Soc. 2005, 26, 2044.

18. Kasahara, A.; Izumi, T:; Ooshima, M. Bull. Chem. Soc. Jpn. 1974 47. 2526. 